\section{DNA-binding domain ancestry}

SIR-We would like to point out a similarity between the DNA-binding domain of the Myb family of proteins and homoeodomain-containing factors. This relationship indicates a common ancestry for the DNA-binding domains of these two classes of eukaryotic nuclear regulatory proteins, and predicts specific roles for parts of the Myb DNA-binding domain.

Genes similar to the c-myb protooncogene have been found in birds, mice, humans, Drosophila melanogaster and plants (Zea mays) (refs 1-6). The region common to the products of all these genes consists of imperfect repeats of 51-53 amino acids; in general there are three such repeats, except in Z.mays, where there are two, and in the two versions of $\mathrm{v}$ Myb (encoded by the AMV and E26 viruses), which are truncated so that most of the first repeat is missing ${ }^{1,7}$. These repeats are responsible for DNA binding ${ }^{8}$, and it is assumed that the rest of the molecule is involved in transactivation of the target gene(s) of Myb (ref. 9).

In the Fig., $a$ shows an alignment of the twenty known Myb repeat sequences, grouped as first, second and third repeats. (Z. mays sequences are best aligned if assigned as repeats 2 and 3.) Eighteen positions are highly conserved for all the sequences, an additional 11 positions being included if the occurrence stringency is lowered to greater than 60 per cent. Sixteen of these positions match with a homoeodomain (HD) consensus if two gaps are introduced ( $b$ in Fig.).

NMR spectroscopy of the homoeodomain from the Antennapedia-gene (Antp) product demonstrates three $a$-helical segments ( $b$ in Fig.) and confirms an earlier prediction that this domain is similar to bacterial repressors, with helices 2 and 3 forming the helixturn-helix structure ${ }^{10}$.

The similarity between Myb and the homoeodomain is reinforced by a prediction of the secondary structure of the Myb repeat $^{3}$ which suggests three $\alpha$-helical

\begin{tabular}{|c|c|c|c|}
\hline$a$ & 1 & . 52 & \\
\hline Drosophila & gfgkrwsksedvlikqlvethg enweiigphfk & drleqquqqrwakvlnpe & \\
\hline $\begin{array}{l}\text { Chicken } \\
\text { Mouse }\end{array}$ & lgkt rwt reedeklkk l veqngtedwkvias f lp & nrtdvqcqhrwqkv lnpe & \\
\hline $\begin{array}{l}\text { Mouse } \\
\text { Human }\end{array}$ & lgkt rwt reedeklkklveqngtddwkvianylp & nrtdvqcqhrwqkvlnpe & First Myb \\
\hline $\begin{array}{l}\text { Human } \\
\text { Human A }\end{array}$ & $\begin{array}{r}\text { rwt reeteklkklveqngtddwkvianylp } \\
\text { wnrvkwt rdetdklkklveghtddwt liashlg }\end{array}$ & $\begin{array}{l}\text { nrtdvqcqhrwqkvinpe } \\
\text { nrsdfqcqhrwqkvlnpe }\end{array}$ & \\
\hline Human B & kckvkwtheeteqlralvrqfgqqdwkfiashfp & nrtdqqcqy rw lrvinpd & \\
\hline Zea mays & vkrgawtskedda la ayvkahgegkwrevpqkag & rcgkscrlrwlnylrpn & \\
\hline Drosophila & likgpwtrdeddmviklvrnfgpkkwtliaryln & grigkqcrerwhnhlnpe & \\
\hline Mouse & likgpwtkeedqrviklvqkygpkrwsviakhlk & grigkqcrerwhnhlnpe & Second Myb \\
\hline Human/chicken & likgpwtkeedqrvielvqkygpkrwsviakhlk & grigkqcrerwhnhlnpe & epe \\
\hline Human A & likgpwtkeedqrvielvqkygpkrwsliakhlk & grigkqcrerwhnhlnpe & \\
\hline Human B & lvkgpwtkeedqkvielvkkygtkqwt liakhlk & grlgrqcrerwhnhlnpe & \\
\hline $\begin{array}{l}\text { Zea mays } \\
\text { Drosophila }\end{array}$ & $\begin{array}{l}\text { irrgnisydeedliirlhrllg nrwsliagrlp } \\
\text { ikktawtekedeiiyqahlelg nqwakiakrlp }\end{array}$ & $\begin{array}{l}\text { grtdneiknywnst lgrr } \\
\text { grtdnaiknhwnstmrrk }\end{array}$ & \\
\hline Chick/mouse/human & vkktswteeedriiyqahkrlg nrwaeiakllp & grtdnaiknhwnstmrrk & repeaf \\
\hline Human A & vkksswteeedrifyeahkrlg nrwaeiakllp & grtansiknhwnstmrrk & \\
\hline Human B & vkkscwteeedriiceahkvlg nrwaeiakmlp & grtdnavknhwnstmerk & \\
\hline :onse & rseed.....t & ..xw....Inps & \\
\hline
\end{tabular}

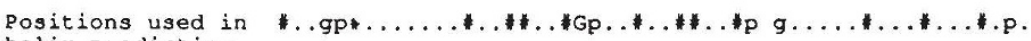
helix prediction

$b$ Predicted Myb
helices

Myb consensus \#.k..WTSeEd...\#\#...G..\$W..IA..L. gRtd.q*..rW...Inp\$ $\begin{array}{cc}\text { Myb aI } & \text { Myb a2 } \\ \text { hһhннннннннн } & \text { йнннннннн }\end{array}$ Myb a3

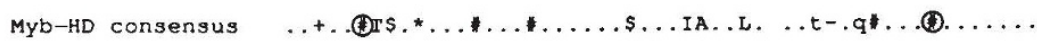

HD consensus rkRgRqtYTRYQtLELEKEFHFNRRRRRIEIAhaL CLtERQIKIWFQNRRMKWKKen 1.

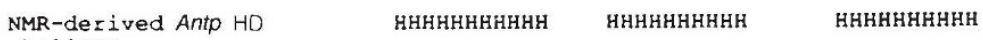

$$
\text { helices }
$$

Bacterial repressor

helix-turn-helix

a, Aligned repeats from Myb-related sequences ${ }^{1-6}$ (single letter amino-acid code), grouped as first, second and third repeats. The derived Myb repeat consensus is shown below (capitals and symbols, $>85 \%$ occurrence in the 20 Myb sequences, lower-case letters, residues which occur in $>60 \%$ of the sequences). Also indicated are the critical positions for helix prediction; conserved hydrophobicity (\#), repeated every 3-4 residues, indicates an amphipathic helix; frequently occurring proline $(P)$ and glycine $(G)$ residues indicate interruptions. $b$, Derived information and comparison of the Myb consensus to the homoeodomain consensus and the bacterial repressor helix-turn-helix motif. The central line of the Fig. indicates the sixteen residues common to the Myb consensus and the homoeodomain (HD) consensus ${ }^{14}$. The extent of the experimentally determined and predicted helical segments are indicated by $\mathrm{H}$ (certainly helical) and $h$ (possibly helical). The lower part of the Fig. shows a consensus for bacterial helixturn-helix motifs derived from a compilation of $37 \lambda$ Cro-like proteins ${ }^{11}$. Residues at conserved positions occur in $>60 \%$ of the sequences. \#, hydrophobic; $\#$, large hydrophobic; \$, charged; +, positive; - , negative; *, E or Q. segments - designated here as Myba13 . On the basis of conserved hydrophobicity and frequently occurring proline and glycine residues, the extent of the $\operatorname{Myb} \alpha$ helices can be estimated, revealing a close correspondence with the three helices of the homoeodomain ${ }^{10}$ (see $b$ in Fig.).

As expected, the two alignment gaps fall within the loops interconnecting the helices. If these extrapolations are correct we would predict that the Myb $\alpha 2$ and Myb $\alpha 3$ helices would form a helix-turnhelix motif, with helix Myb $\alpha 3$ making direct contact with bases in the DNA. Furthermore, the most conserved positions $^{11}$ in the bacterial repressor helixturn-helix motif (which is related to the homoeodomain) align well with positions in the Myb consensus, especially those used in helix prediction (see $b$ in Fig.). Applying a statistical algoritham derived from the bacterial sequences used to generate the helix-turn-helix consensus (ref. 11, with the modification suggested in ref. 12) to the homoeodomain consensus helix2-turn-helix 3 region and the predicted Myb $\alpha 2$-turn-Myb $\alpha 3$ domain of the third repeat in chicken Myb (discounting the single insertion), gave scores of 1,363 and 1,358 , respectively, compared with 1,675 for the $\lambda$ Cro repressor and 1,336 for the $\lambda \mathrm{cI}$ repressor.

Myb sequences within a particular repeat grouping are most highly related at the $\mathrm{C}$ terminus, in the region of helix Myba3 (as expected if this is the intimate DNA contact point). A 'patch' of positive amino acids, as seen at the end of helix 3 in the homoeodomain, can also be seen at the end of the helix Myba 3 in the repeat 3 sequences but not at the equivalent position in repeats 1 and 2.

A specific role for tryptophans in the Myb DNA-binding structure has been recently suggested $^{13}$; it now seems likely that this may involve their hydrophobic character as, by analogy to the bacterial repressors, the tryptophans would be within a hydrophobic core.

JONATHAN FRAMPTON

ACHIM LEUTZ

TOBY J. GIBSON

THOMAS GRAF

European Molecular Biology Laboratory,

Postfach 10.2209,

Meyerhofstrasse 1,

6900 Heidelberg, FRG

1. Rosson, D. \& Reddy, E.P Nature 319, 604-606 (1986).

2. Gerondakis, S. \& Bishop, J.M. Molec. cell Biol. 6, 36773684 (1986).

3. Gonda, T.J. et al. EMBO J. 4, 2003-2008 (1985),

4. Nomura, N. et al. Nucleic Acids Res. 16, 11075 (1988).

5. Katzen, A.L., Kornberg, T.B. \& Bishop, J.M. Cell 41, 449456 (1985)

6. Paz-Ares, J. et al. EMBO J. 6, 3553-3558 (1987)

7. Nunn, M.F. etal. Nature 306, 391-395 (1983).

8. Klempnauer, K-H. \& Sippel, A.E. EMBO J. 6, 2719-2725 (1987).

9. Nishina, Y. et al. Nucleic Acids Res. 17, 107 (1989) 10. Otting, G. et al. EMBO J. 7, 4305-4309 (1988)

11. Dodd, I.B. \& Egan, J.B. J. molec. Biol. 194, 557 (1987).

12. Yudkin, M.D. Protein Eng. 1, 371-372 (1987).

13. Anton, I.A. \& Frampton, J. Nature 336, 719 (1988).

14. Muller, M.M. et al. Nature 336, 544-551 (1988). 